\title{
Self-intersections for the Willmore flow
}

\author{
Uwe F. Mayer and Gieri Simonett
}

\begin{abstract}
We prove that the Willmore flow can drive embedded surfaces to self-intersections in finite time.
\end{abstract}

\section{Introduction}

In this paper we consider the Willmore flow in three space dimensions. We prove that embedded surfaces can be driven to a self-intersection in finite time. This situation is in strict contrast to the behavior of hypersurfaces under the mean curvature flow, where the maximum principle prevents self-intersections, but very much analogous to the surface diffusion flow.

The Willmore flow is a geometric evolution law in which the normal velocity of a moving surface equals the Laplace-Beltrami of the mean curvature plus some lower order terms. More precisely, we assume in the following that $\Gamma_{0}$ is a closed compact immersed and orientable surface in $\mathbb{R}^{3}$. Then the Willmore flow is governed by the law

$$
V(t)=\Delta_{\Gamma(t)} H_{\Gamma(t)}+2 H_{\Gamma(t)}\left(H_{\Gamma(t)}^{2}-K_{\Gamma(t)}\right), \quad \Gamma(0)=\Gamma_{0} .
$$

Here $\Gamma=\{\Gamma(t) ; t \geq 0\}$ is a family of smooth immersed orientable surfaces, $V(t)$ denotes the velocity of $\Gamma$ in the normal direction at time $t$, while $\Delta_{\Gamma(t)}, H_{\Gamma(t)}$, and $K_{\Gamma(t)}$ stand for the Laplace-Beltrami operator, the mean curvature, and the Gauß curvature of $\Gamma(t)$, respectively.

The evolution law (1.1) does not depend on the local choice of the orientation. However, if $\Gamma(t)$ is embedded and encloses a region $\Omega(t)$ we always choose the outer normal, so that $V(t)$ is positive if $\Omega(t)$ grows, and so that $H_{\Gamma(t)}$ is positive if $\Gamma(t)$ is convex with respect to $\Omega(t)$.

Any equilibrium of (1.1), that is, any closed smooth surface that satisfies the equation

$$
\Delta H+2 H\left(H^{2}-K\right)=0
$$

is called a Willmore surface $[18$, p. 282]. There has been much interest over the last years in characterizing Willmore surfaces, see for instance $[15,18]$ and the references cited therein. Willmore surfaces arise as the critical points of the 
functional

$$
W(f):=\int_{f(M)} H^{2} d S,
$$

see [18, Section 7.4]. Here, $M$ denotes a smooth closed orientable surface and $f: M \rightarrow \mathbb{R}^{3}$ is a smooth immersion of $M$ into $\mathbb{R}^{3}$. Associated with this functional is a variational problem: Given a smooth closed orientable surface $M_{g}$ of genus $g$ determine the infimum $W\left(M_{g}\right)$ of $W(f)$ over all immersions $f: M_{g} \rightarrow \mathbb{R}^{3}$ and classify all manifolds $f\left(M_{g}\right)$ which minimize $W$. We refer to $[4,8,14,15,17,18]$ and the references therein for more details and interesting results.

The Willmore flow is the $L^{2}$-gradient flow for the functional (1.3) on the moving boundary, see for example [7], and also [10] for related work on gradient flows. Thus the Willmore flow has the distinctive property that it evolves surfaces in such a way as to reduce the total quadratic curvature. To be more precise, we show that the flow decreases the total quadratic curvature for any $C^{2+\beta}$ initial surface $\Gamma_{0}$.

Proposition 1. Let $0<\beta<1$ and let $\Gamma_{0}$ be a closed compact immersed orientable surface that is $C^{2+\beta}$-smooth. Then

$$
\int_{\Gamma(t)} H^{2}(t) d \mu \leq \int_{\Gamma_{0}} H^{2}(0) d \mu, \quad 0 \leq t \leq T,
$$

where $[0, T]$ denotes the interval of existence guaranteed in the existence theorem of [16], and where $H(t)$ denotes the mean curvature of $\Gamma(t)$.

To the best of our knowledge, the result of Proposition 1 is new (under the given assumptions).

Next we show that the flow can force $\Gamma(t)$ to lose embeddedness in order to decrease the total quadratic curvature.

Theorem 2. Let $0<\beta<1$ be fixed.

There exist a closed embedded surface $\Sigma_{0} \in C^{2+\beta}$, a constant $T_{0}>0$, numbers $t_{0}, t_{1} \in\left(0, T_{0}\right]$ with $t_{0}<t_{1}$, and a $C^{2+\beta}{ }_{-n e i g h b o r h o o d ~} U_{0}$ of $\Sigma_{0}$ such that

(a) the Willmore flow (1.1) has a unique classical solution $\Gamma=\left\{\Gamma(t) ; t \in\left[0, T_{0}\right]\right\}$ for all $\Gamma_{0} \in U_{0}$,

(b) $\Gamma(t)$ ceases to be embedded for every $t \in\left(t_{0}, t_{1}\right)$ and every $\Gamma_{0} \in U_{0}$.

(c) each surface $\Gamma(t)$ is of class $C^{\infty}$ for $t \in\left(0, T_{0}\right]$ and smooth in $t \in\left(0, T_{0}\right)$.

It should be noted that the neighborhood $U_{0}$ of Theorem 2 also contains $C^{\infty}$ surfaces that will be driven to a self-intersection in finite time. Our approach relies on results and techniques in $[6,12,16]$, and we follow closely the original argument in [12].

Lastly we mention that numerical simulations [13] seem to indicate that the Willmore flow can drive immersed surfaces to topological changes in finite time. 


\section{The mathematical setting}

We first introduce some notations. Given an open set $U \subset \mathbb{R}^{3}$, let $h^{s}(U)$ denote the little Hölder spaces of order $s>0$, that is, the closure of $B U C^{\infty}(U)$ in $B U C^{s}(U)$, the latter space being the Banach space of all bounded and uniformly Hölder continuous functions of order $s$. If $\Sigma$ is a (sufficiently) smooth submanifold of $\mathbb{R}^{3}$ then the spaces $h^{s}(\Sigma)$ are defined by means of a smooth atlas for $\Sigma$. It is known that $B U C^{t}(\Sigma)$ is continuously embedded in $h^{s}(\Sigma)$ whenever $t>s$. In the following, we assume that $\Sigma$ is a smooth compact closed immersed oriented surface in $\mathbb{R}^{3}$. Let $\nu$ be the unit normal field on $\Sigma$ commensurable with the chosen orientation. Then we can find $a>0$ and an open covering $\left\{U_{l} ; l=1, \ldots, m\right\}$ of $\Sigma$ such that

$$
X_{l}: U_{l} \times(-a, a) \rightarrow \mathbb{R}^{3}, \quad X_{l}(s, r):=s+r \nu(s),
$$

is a smooth diffeomorphism onto its image $\mathcal{R}_{l}:=\operatorname{im}\left(X_{l}\right)$, that is,

$$
X_{l} \in \operatorname{Diff}^{\infty}\left(U_{l} \times(-a, a), \mathcal{R}_{l}\right), \quad 1 \leq l \leq m .
$$

This can be done by selecting the open sets $U_{l} \subset \Sigma$ in such a way that they are embedded in $\mathbb{R}^{3}$ instead of only immersed, and then taking $a>0$ sufficiently small so that each of the $U_{l}$ has a tubular neighborhood of radius $a$. It follows that $\mathcal{R}:=\cup \mathcal{R}_{l}$ consists of those points in $\mathbb{R}^{3}$ with distance less than $a$ to $\Sigma$. Let $\beta \in(0,1)$ be fixed. Then we choose numbers $\alpha, \beta_{1} \in(0,1)$ with $\alpha<\beta_{1}<\beta$. Let

$$
W:=\left\{\rho \in h^{2+\beta_{1}}(\Sigma) ;\|\rho\|_{\infty}<a\right\} .
$$

Given any $\rho \in W$ we obtain a compact oriented immersed manifold $\Gamma_{\rho}$ of class $h^{2+\beta_{1}}$ by means of the following construction:

$$
\Gamma_{\rho}:=\bigcup_{l=1}^{m} \operatorname{Im}\left(X_{l}: U_{l} \rightarrow \mathbb{R}^{3},\left[s \mapsto X_{l}(s, \rho(s))\right]\right) .
$$

Thus $\Gamma_{\rho}$ is a graph in normal direction over $\Sigma$ and $\rho$ is the signed distance between $\Sigma$ and $\Gamma_{\rho}$. On the other hand, every compact immersed oriented manifold $\Gamma$ that is a smooth graph over $\Sigma$ and that is contained in $\mathcal{R}$ can be obtained in this way. For convenience we introduce the mapping

$$
\theta_{\rho}: \Sigma \rightarrow \Gamma_{\rho}, \quad \theta_{\rho}(s):=X_{l}(s, \rho(s)) \text { for } s \in U_{l}, \quad \rho \in W .
$$

It follows that $\theta_{\rho}$ is a well-defined global $\left(2+\beta_{1}\right)$-diffeomorphism from $\Sigma$ onto $\Gamma_{\rho}$. The Willmore flow (1.1) can now be expressed as an evolution equation for the distance function $\rho$ over the fixed reference manifold $\Sigma$,

$$
\partial_{t} \rho=G(\rho), \quad \rho(0)=\rho_{0} .
$$

Here $G(\rho):=L_{\rho} \theta_{\rho}^{*}\left(\Delta_{\Gamma_{\rho}} H_{\Gamma_{\rho}}+2 H_{\Gamma_{\rho}}\left(H_{\Gamma_{\rho}}^{2}-K_{\Gamma_{\rho}}\right)\right)$ for $\rho \in h^{4+\alpha}(\Sigma) \cap W$, while $\Delta_{\Gamma_{\rho}}, H_{\Gamma_{\rho}}$, and $K_{\Gamma_{\rho}}$ are the Laplace-Beltrami operator, the mean curvature, and 
the Gauss curvature of $\Gamma_{\rho}$, respectively, and $L(\rho)$ is a factor that comes in by calculating the normal velocity in terms of $\rho$, see [6] for more details. We are now ready to state the following existence result for solutions of (2.3).

Proposition 2.1. Let $\sigma \in W$ be given.

(a) There exist a positive constant $T_{0}>0$ and a neighborhood $W_{0} \subset W$ of $\sigma$ in $h^{2+\beta_{1}}(\Sigma)$ such that $(2.3)$ has a unique solution

$$
\rho\left(\cdot, \rho_{0}\right) \in C\left(\left[0, T_{0}\right], W\right) \cap C^{\infty}\left(\left(0, T_{0}\right) \times \Sigma\right) \text { for every } \rho_{0} \in W_{0} .
$$

(b) The map $\left[\left(t, \rho_{0}\right) \mapsto \rho\left(t, \rho_{0}\right)\right]$ defines a smooth local semiflow on $W_{0}$.

(c) $\rho\left(\cdot, \rho_{0}\right) \in C\left(\left[0, T_{0}\right], h^{4+\alpha}(\Sigma)\right) \cap C^{1}\left(\left[0, T_{0}\right], h^{\alpha}(\Sigma)\right)$ for all $\rho_{0} \in h^{4+\alpha}(\Sigma) \cap W_{0}$.

Proof. (a) and (b) follow from [16, Proposition 2.2]. Moreover, [16, Lemma 2.1] shows that the mapping $[\rho \mapsto G(\rho)]: h^{4+\alpha}(\Sigma) \cap W \rightarrow h^{\alpha}(\Sigma)$ is smooth and that the derivative is given by $G^{\prime}(\rho)=P(\rho)+B(\rho)$, where

$$
P(\rho) \in L\left(h^{4+\alpha}(\Sigma), h^{\alpha}(\Sigma)\right), \quad B(\rho) \in L\left(h^{2+\alpha}(\Sigma), h^{\alpha}(\Sigma)\right), \quad \rho \in h^{4+\alpha}(\Sigma) \cap W .
$$

In the following we fix $\rho \in h^{4+\alpha}(\Sigma) \cap W$. [16, Lemma 2.1] also shows that $P(\rho)$ generates a strongly continuous analytic semigroup on $h^{\alpha}(\Sigma)$. A well-known perturbation result, see [1, Theorem I.1.3.1], then implies $G^{\prime}(\rho) \in L\left(h^{4+\alpha}(\Sigma), h^{\alpha}(\Sigma)\right)$ also generates a strongly continuous analytic semigroup on $h^{\alpha}(\Sigma)$. It is known that the little Hölder spaces are stable under the continuous interpolation method $[1,2,5,9]$. Therefore, the spaces $\left(h^{4+\alpha}(\Sigma), h^{\alpha}(\Sigma)\right)$ form a pair of maximal regularity for $G^{\prime}(\rho)$, see [1, Theorem III.3.4.1] or $[2,5,9]$. Part (c) follows now from maximal regularity results, for instance [2, Theorem 2.7].

\section{The proof of Proposition 1}

We first note that any function in $C^{2+\beta}$ is also in $h^{2+\beta_{1}}$ for $\beta_{1} \in(0, \beta)$. Let $\Gamma_{0}$ be a given surface in $\mathbb{R}^{3}$ that satisfies the assumptions of Proposition 1 . We can find a smooth surface $\Sigma$ as in Section 2 and a function $\rho_{0} \in W$ such that $\Gamma_{0}=\Gamma_{\rho_{0}}$, where $\Gamma_{\rho_{0}}$ is defined in (2.2). According to Proposition 2.1(a) there exists a number $T=T\left(\rho_{0}\right)>0$ such that equation (2.3) has a unique solution $\rho\left(\cdot, \rho_{0}\right)$ with the smoothness properties stated in the proposition. It follows from the construction in Section 2 that the family $\Gamma:=\{\Gamma(t) ; 0 \leq t \leq T\}$, where $\Gamma(t):=\Gamma_{\rho(t)}$ for $0 \leq t \leq T$, is the unique classical solution for the Willmore flow (1.1). In particular, we conclude that

$$
\left[t \mapsto \int_{\Gamma(t)} H^{2}(t) d \mu\right] \in C^{\infty}((0, T), \mathbb{R})
$$


Given $x \in \Gamma(t)$, let $\left\{z(\tau, x) \in \mathbb{R}^{3} ; \tau \in(-\varepsilon, \varepsilon)\right\}$ be an orthogonal flow line through $x$, that is, $z(\cdot, x)$ satisfies

$$
\begin{aligned}
& z(\tau, x) \in \Gamma(t+\tau) \text { for } \tau \in(-\varepsilon, \varepsilon), \\
& \dot{z}(\tau)=(V N)(t+\tau, z(\tau)) \text { for } \tau \in(-\varepsilon, \varepsilon), z(0)=x,
\end{aligned}
$$

where $N(t, \cdot)$ denotes the unit normal field on $\Gamma(t)$, and $V(t, \cdot)$ is the normal velocity of $\Gamma(t)$. A proof for the existence of a unique trajectory $\left\{z(\tau, x) \in \mathbb{R}^{3} ; \tau \in\right.$ $(-\varepsilon, \varepsilon)\}$ with the above properties can for instance be found in [11, Lemma 2.1]. For further use we introduce the manifold $\mathcal{M}:=\bigcup_{t \in(0, T)}\{t\} \times \Gamma(t)$. Given any smooth function $u$ on $\mathcal{M}$ we define

$$
\frac{d}{d t} u(t, x):=\left.\frac{d}{d \tau} u(t+\tau, z(\tau, x))\right|_{\tau=0}, \quad(t, x) \in \mathcal{M} .
$$

The following differentiation rule is well-known in differential geometry,

$$
\frac{d}{d t} \int_{\Gamma(t)} u(t, x) d \mu(x)=\int_{\Gamma(t)} \frac{d}{d t} u(t, x) d \mu(x)+2 \int_{\Gamma(t)}(u H V)(t, x) d \mu(x) .
$$

Let $(t, x) \in \mathcal{M}$ be fixed and let $\{z(\tau, x) ; \tau \in(-\varepsilon, \varepsilon)\}$ be a flow line trough $x$. Then one can show that

$$
\left.\frac{d}{d \tau} H^{2}(t+\tau, z(\tau, x))\right|_{\tau=0}=-H\left[\Delta_{\Gamma(t)} V+\left(4 H^{2}-2 K\right) V\right](t, x),
$$

see for instance [18, Section 7.4]. If follows from (3.1)-(3.2), from the divergence theorem, and from (1.1) that

$$
\frac{d}{d t} \int_{\Gamma(t)} H^{2}(t) d \mu=-\int_{\Gamma(t)}\left[\Delta H+2 H\left(H^{2}-K\right)\right] V d \mu \leq 0 .
$$

This is true for any $t \in(0, T)$. The mean value theorem now implies that

$$
\int_{\Gamma(t)} H^{2}(t) d \mu-\int_{\Gamma(\tau)} H^{2}(\tau) d \mu \leq 0 \quad \text { for } 0<\tau \leq t<T .
$$

Taking the limit as $\tau \rightarrow 0$ and using that $\left[\tau \mapsto \int_{\Gamma(\tau)} H^{2}(\tau) d \mu\right] \in C([0, T], \mathbb{R})$, see Proposition 2.1(b), yields the assertion of Proposition 1.

\section{The proof of Theorem 2}

In order to provide a proof of Theorem 2 we now choose $\Sigma$ to be any smooth compact closed immersed orientable surface in $\mathbb{R}^{3}$ such that its image contains the flat 2-dimensional disk $U:=\left\{(s, 0) \in \mathbb{R}^{2} \times \mathbb{R} ;|s| \leq 1\right\}$ twice, and with opposite 
orientations. To be precise, let $i: \Sigma \rightarrow \mathbb{R}^{3}$ be the immersion under consideration, then we ask that

$$
i^{-1}(U)=U^{+} \cup U^{-}
$$

with $U^{+} \cap U^{-}=\emptyset$ and both $U^{+}$and $U^{-}$are flat 2-dimensional disks of radius 1 . Additionally we ask that $\Sigma \backslash\left(U^{+} \cup U^{-}\right)$is embedded in $\mathbb{R}^{3}$. Identifying $U^{+}$for the moment with its image $U$ we ask that the normal on $U^{+}$points upwards, that is, $\left.\nu(\cdot)\right|_{U^{+}}=e_{3}$, the $3^{\text {rd }}$ basis vector of $\mathbb{R}^{3}$. It follows that $\left.\nu(\cdot)\right|_{U^{-}}=-e_{3}$.

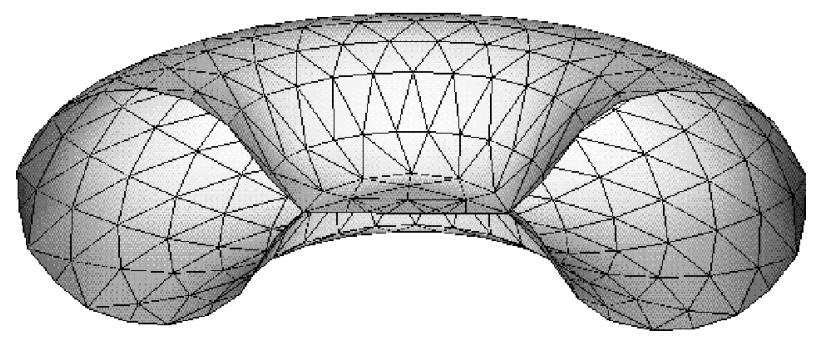

Fig. 1 This is a possible choice of $\Sigma$, cut in halves.

Let $W$ be as in (2.1) and let $\sigma \in h^{4+\alpha} \cap W$ locally be radially symmetric with regards to the centers of $U^{ \pm}$. This implies $\partial_{j} \sigma(0)=0$ for $j=1,2$. Observe that $\theta_{\sigma}(s)=(s, \pm \sigma(s))$ (these are coordinates in $\mathbb{R}^{3}$ ) for $s \in U^{ \pm}$and that $\theta_{\sigma}: U^{ \pm} \rightarrow$

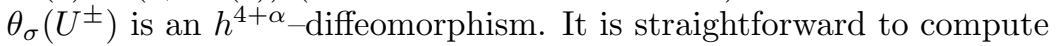

$$
\left.G(\sigma)\right|_{U^{ \pm}}:=\left.L(\sigma) \theta_{\sigma}^{*}\left(\Delta_{\Gamma_{\sigma}} H_{\Gamma_{\sigma}}+2 H_{\Gamma_{\sigma}}\left(H_{\Gamma_{\sigma}}^{2}-K_{\Gamma_{\sigma}}\right)\right)\right|_{U^{ \pm}}
$$

in local coordinates, yielding

$$
\begin{aligned}
\left.2 G(\sigma)\right|_{U^{ \pm}}(0)= & -\Delta^{2} \sigma(0)+\sum_{j, k=1}^{2}\left(\partial_{j} \partial_{k} \sigma(0)\right)^{2} \Delta \sigma(0) \\
& +2 \sum_{j, k, l=1}^{2} \partial_{j} \partial_{k} \sigma(0) \partial_{j} \partial_{l} \sigma(0) \partial_{k} \partial_{l} \sigma(0),
\end{aligned}
$$

where $\Delta$ is the Laplacian in Euclidean coordinates of $\mathbb{R}^{2}$ (see [6, Section 2] for more details). Because of the radial symmetry of $\sigma$ we have $H_{\Gamma_{\sigma}}^{2}=K_{\Gamma_{\sigma}}$ at the center of the disks $U^{ \pm}$, so that lower order term $\theta_{\sigma}^{*}\left(2 H_{\Gamma_{\sigma}}\left(H_{\Gamma_{\sigma}}^{2}-K_{\Gamma_{\sigma}}\right)\right)$ vanishes at the center of $U^{ \pm}$. We will now specify one more property of $\sigma$. We choose $r>0$ small and we require that $\sigma(s)=|s|^{4}$ for $s \in U_{r}^{ \pm}=\left\{s \in U^{ \pm} ;|s|<r\right\}$. If $r$ is small enough then this is compatible with $\sigma \in h^{4+\alpha}(\Sigma) \cap W$. We conclude that

$$
\left.G(\sigma)\right|_{U^{ \pm}}(0)=-16<0 .
$$


It follows from Proposition 2.1 that the evolution equation (2.3) with initial value $\rho(0)=\sigma$ has a unique solution

$$
\rho(\cdot, \sigma) \in C\left(\left[0, T_{0}\right], h^{4+\alpha}(\Sigma)\right) \cap C^{1}\left(\left[0, T_{0}\right], h^{\alpha}(\Sigma)\right) .
$$

Next we consider the restriction $\rho^{ \pm}(t, \sigma)$ on $U^{ \pm}$of the function $\rho(t, \sigma)$, that is, $\rho^{ \pm}(t, \sigma):=\left.\rho(t, \sigma)\right|_{U^{ \pm}}$for $0 \leq t \leq T_{0}$, and we set $d^{ \pm}(t):=\rho^{ \pm}(t, \sigma)(0)$, to track the position of the center. It follows from (4.2) that $d^{ \pm} \in C^{1}\left(\left[0, T_{0}\right]\right)$. Moreover, using the local character of $G$, we conclude that $d^{ \pm}$satisfies the equation

$$
\left(d^{ \pm}\right)^{\prime}(t)=\left.G(\rho(t, \sigma))\right|_{U^{ \pm}}(0) \quad \text { for } \quad 0 \leq t \leq T_{0}, \quad d^{ \pm}(0)=0 .
$$

Equations (4.1)-(4.3) and the mean value theorem yield

$$
d^{ \pm}(t)=-M t+\left(\int_{0}^{1}\left(\left(d^{ \pm}\right)^{\prime}(\tau t)-\left(d^{ \pm}\right)^{\prime}(0)\right) d \tau\right) t,
$$

where $M:=16$. It follows from (4.4) that there exists a positive constant $\mu>0$ and an interval $\left(t_{0}, t_{1}\right) \subset\left(0, T_{0}\right]$ such that $\rho^{ \pm}(t, \sigma)(0)=d^{ \pm}(t) \leq-\mu$ for $t \in\left(t_{0}, t_{1}\right)$. By Proposition 2.1(b) we can find a function $\sigma_{0} \in W_{0}$ such that $\Sigma_{0}:=\Gamma_{\sigma_{0}}$ is embedded and such that $\Gamma(t):=\Gamma_{\rho\left(t, \sigma_{0}\right)}$ is immersed for at least $t \in\left(t_{0}, t_{1}\right)$. By employing Proposition 2.1(b) once more we conclude there is a neighborhood $W\left(\sigma_{0}\right) \subset W_{0}$ of $\sigma_{0}$ in $h^{2+\beta_{1}}(\Sigma)$ such that $\Gamma_{\rho_{0}}$ is still embedded, whereas $\Gamma_{\rho\left(t, \rho_{0}\right)}$ is immersed for $t \in\left(t_{0}, t_{1}\right)$ and all $\rho_{0} \in W\left(\sigma_{0}\right)$. We note that $C^{2+\beta}(\Sigma)$ is contained in $h^{2+\beta_{1}}(\Sigma)$ with continuous injection $j: C^{2+\beta}(\Sigma) \rightarrow h^{2+\beta_{1}}(\Sigma)$. Hence $U_{0}:=j^{-1}\left(W\left(\sigma_{0}\right)\right)$ is a $C^{2+\beta}$-neighborhood of $\sigma_{0}$ and Theorem 2 follows by choosing $\Sigma_{0}:=\Gamma_{\sigma_{0}}$ and $\Gamma_{0}:=\Gamma_{\rho_{0}}$ for $\rho_{0} \in U_{0}$.

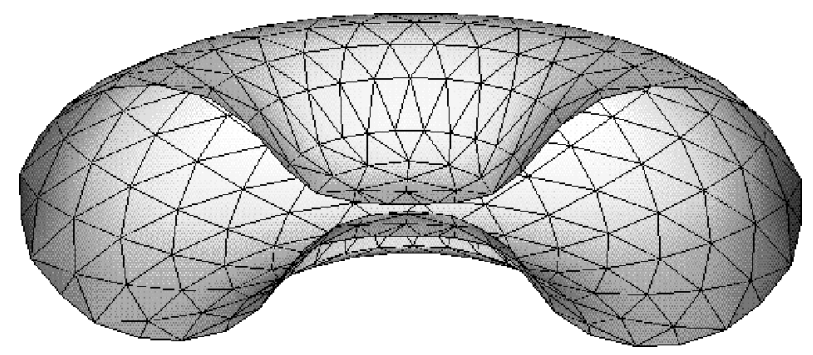

Fig. 2 This is half of $\Gamma_{0}$, a surface that loses embeddedness and becomes immersed. The gap might have to be much smaller than depicted.

Remark 4.2. The following is the essence of the construction: $\Gamma_{\sigma}$ is an immersed surface such that its image contains two opposing fourth-order paraboloids touching only at the vertex. The global symmetry of $\Gamma_{\sigma}$ is irrelevant, we only need the local symmetry at the center. Locally we can compute the initial velocity of $\Gamma_{\sigma}$, 
and it is such as to create an overlapping of the fourth-order paraboloids. A continuity argument then guarantees the same behavior for nearby embedded surfaces, which do exist by construction of $\Gamma_{\sigma}$. We have chosen a fourth-order paraboloid in order to facilitate the computation of $\left.G(\sigma)\right|_{U_{ \pm}}$. Any other configuration that produces the same sign as in (4.1) will work as well.

\section{References}

[1] H. Amann, Linear and quasilinear parabolic problems. Vol. I, Birkhäuser, Basel, 1995.

[2] S.B. Angenent, Nonlinear analytic semiflows, Proc. Roy. Soc. Edinburgh Sect. A 115 (1990), 91-107.

[3] R. Bryant, A duality theorem for Willmore surfaces, J. Diff. Geom. 20 (1984), 23-53.

[4] B.-Y. Chen, On a variational problem on hypersurfaces, J. London Math. Soc. 2 (1973), 321-325.

[5] G. DaPrato and P. Grisvard, Equations d'évolution abstraites nonlinéaires de type parabolique, Ann. Mat. Pura Appl. (4) 120 (1979), 329-396.

[6] J. Escher, U.F. Mayer, and G. Simonett, The surface diffusion flow for immersed hypersurfaces, SIAM J. Math. Anal. 29 (1998), 1419-1433.

[7] E. Kuwert and R. Schätzle, Gradient flow for the Willmore functional, Comm. Anal. Geom. 10 (2002), 307-339.

[8] R. Kusner, Estimates for the biharmonic energy on unbounded planar domains, and the existence of surfaces of every genus that minimize the squaredmean-curvature integral in Elliptic and parabolic methods in geometry (Minneapolis, MN, 1994), A K Peters, Wellesley, MA, 1996, 67-72.

[9] A. Lunardi, Analytic semigroups and optimal regularity in parabolic problems, Birkhäuser, Basel, 1995.

[10] U.F. Mayer, A numerical scheme for free boundary problems that are gradient flows for the area functional, Europ. J. Appl. Math. 11 (2000), 61-80.

[11] U.F. Mayer and G. Simonett, Classical solutions for diffusion-induced grainboundary motion, J.Math. Anal. Appl. 234 (1999), 660-674.

[12] U.F. Mayer and G. Simonett, Self-intersections for the surface diffusion and the volume preserving mean curvature flow, Differential Integral Equations 13 (2000), 1189-1199. 
[13] U.F. Mayer and G. Simonett, A numerical scheme for axisymmetric solutions of curvature driven free boundary problems, with applications to the Willmore flow, Interfaces and Free Boundaries 4 (2002), 1-22.

[14] U. Pinkall, Hopf tori in $S^{3}$, Invent. Math. 81 (1985), 379-386.

[15] U. Pinkall and I. Sterling, Willmore surfaces, Math. Intelligencer 9 (1987), $38-43$.

[16] G. Simonett, The Willmore flow near spheres, Differential Integral Equations 14 (2001), 1005-1014.

[17] L. Simon, Existence of surfaces minimizing the Willmore functional, Comm. Anal. Geom. 1 (1993), 281-326.

[18] T.J. Willmore, Riemannian Geometry, Claredon Press, Oxford, 1993.

387 Trailview Road, Encinitas, CA 92024, U.S.A.

mayer@math.utah.edu

Department of Mathematics, Vanderbilt University, Nashville, TN 37240, U.S.A. simonett@math.vanderbilt.edu 